\title{
Procesos de cambio en las universidades nacionales: un estudio sobre los diseños institucionales de la Carrera Docente / Académica en Argentina
}

Change processes in national universities:

a study on the institutional designs of the Teaching / Academic Career in Argentina

Daniela Atairo

https://orcid.org/0000-0002-2887-3407

atairodaniela@gmail.com

Facultad de Humanidades y Ciencias de la Educación

Universidad Nacional de la Plata | Argentina

\author{
Laura Rovelli \\ https://orcid.org/0000-0001-7059-149X \\ laurarovelli@gmail.com \\ Facultad de Humanidades y Ciencias de la Educación \\ Universidad Nacional de la Plata | Argentina
}

\section{RESUMEN}

El artículo presenta un estudio exploratorio sobre regímenes de Carrera Docente/Académica (en adelante, $\mathrm{CD} / \mathrm{CA}$ ) en una muestra seleccionada intencionalmente entre las universidades públicas de la Argentina. La perspectiva teórica se beneficia de algunas herramientas conceptuales del análisis de las políticas públicas, al recuperar la complejidad en el proceso y la diversidad de sentidos en la implementación de políticas a la vez, que procura identificar cambios en los diseños de CD/CA desde un plano institucional. Un argumento general de la investigación sostiene que los diseños analizados presentan diversidad en sus formas como resultado de las configuraciones organizacionales específicas, a la vez que comparten ciertas tendencias comunes en el diseño de la CD/CA, lo que expresa valores y creencias nuevas en torno a la regulación de la profesión docente.

\section{ABSTRACT}

The article presents an exploratory study on Teaching / Academic Career regimes (hereinafter, $C D$ / CA) in a sample intentionally selected from public universities in Argentina. The theoretical perspective benefits from some conceptual tools of public policy analysis, by recovering the complexity of the process and the diversity of meanings in the implementation of policies at the same time, which seeks to identify changes in CD / CA designs from a institutional level A general argument from the research argues that the designs analyzed have diversity in their forms as a result of specific organizational configurations, while sharing certain common trends in the design of the $C D$ / $C A$, which expresses new values and beliefs in around the regulation of the teaching profession.

PALABRAS CLAVE Universidades; Carrera Docente/Académica; Evaluación docente; Cambio institucional.
KEY WORDS

Universities; Teaching / Academic Career; Teacher evaluation; Institutional changes 


\section{INTRODUCCIÓN:}

En las últimas cuatro décadas, los circuitos de evaluación de la actividad de los docentes universitarios en la Argentina se configuran de manera heterogénea. En el marco de la normalización universitaria, se desarrolla un complejo entramado de estatutos y reglamentos de concursos elaborados desde las respectivas universidades nacionales. A partir de las décadas de 1990 y 2000, se suman las racionalidades y los criterios de los programas de las agencias gubernamentales del sector de Educación Superior y de Ciencia y Tecnología, orientadas a promover y orientar de manera directa o indirecta la actividad a través de distintos programas e instrumentos. A su vez, entre los años 2004 y 2015, la recomposición y homologación salarial de los docentes universitarios es acompañada por lineamientos de desarrollo y fortalecimiento de la carrera académica, como parte de la ampliación de una agenda de regulación de las relaciones laborales-académicas desde el ámbito paritario (Pérez y Solanas, 2015). A lo anterior se agrega la homologación en 2015 del primer Convenio Colectivo de Trabajo (CCT) para los docentes de las Instituciones Universitarias Nacionales por parte del Poder Ejecutivo Nacional (Dto. n. $1246 / 15)$, que garantiza -entre otros derechos- el acceso y la permanencia de los trabajadores a la Carrera Docente en las universidades nacionales del país (Rovelli, De la Fare y Lenz, 2016).

Desde entonces distintas universidades nacionales procuran regular de manera general la actividad de su personal docente, a través de la implementación de regímenes de Carrera Docente/Académica (en adelante, CD/CA). Con ello, buscan garantizar la estabilidad laboral de los docentes a partir de la evaluación periódica e integral de diversas actividades, entre las que destaca la docencia. Un primer grupo de regímenes de CD/CA surge en la primera mitad de la década de 1990, esas normativas luego suman algunas modificaciones en la primera década de este siglo. A partir de esos años, proliferan reglamentaciones en varias instituciones, mientras que otras casas de estudio creadas con posterioridad al 2007 inician su actividad institucional con normativas específicas sobre CD/CA o reglamentos docentes que las incluyen.

En ese escenario, el artículo presenta los primeros resultados de un estudio exploratorio centrado en los diseños institucionales de la CD/ CA en un conjunto de universidades nacionales de la Argentina. Desde en el análisis de las políticas públicas, la perspectiva teórica recupe- 
ra la complejidad en el proceso y la diversidad de sentidos involucrados en la implementación de políticas a la vez, que procura identificar cambios en los diseños de CD/CA desde un plano institucional. Para ello, se seleccionan algunos casos de estudio y se analizan los distintos diseños institucionales y sus implicancias. Uno de los argumentos centrales de la investigación sostiene que los diseños analizados presentan diversidad en sus formas como resultado de las configuraciones organizacionales específicas, a la vez que comparten ciertas tendencias comunes en el diseño de la CD/CA, lo que expresa valores y creencias nuevas en torno a la regulación de la profesión docente.

En adelante, el escrito se organiza en tres apartados. En el primero, se delinea el enfoque y la estrategia metodológica del estudio. En la segunda sección se despliega el análisis de las cinco dimensiones seleccionadas para analizar los diseños institucionales de la CD/CA: acceso, permanencia y promoción; funciones, categorías y perfiles docentes; conformación de las comisiones evaluadoras y las modalidades y dispositivos de evaluación. A su vez, comparamos las tendencias en esas dimensiones con los lineamientos generales del Convenio Colectivo de Trabajo (CCT). Por último, en las consideraciones finales se sistematizan algunas tendencias y se abren algunos interrogantes sobre el objeto de estudio en el contexto actual.

\section{ENFOQUE TEÓRICO Y ESTRATEGIA METODOLÓGICA}

El estudio de la dimensión del cambio en las políticas abre un conjunto de interrogantes variado acerca de cuándo y dónde acontece, cómo evaluarlo, cuál es su origen y por qué ocurre, pudiendo así adoptar distinto tratamiento de acuerdo con la priorización de abordajes teóricos y epistemológicos distintos (Fontaine, 2015).

En este trabajo, nos preguntamos por el sentido del cambio desde una perspectiva teórica neoinstitucional, la que se orienta a desentrañar la importancia de las instituciones en el desenvolvimiento de las políticas públicas (Fontaine, 2015). Al respecto, recuperamos el concepto de isomorfismo institucional elaborado por Di Maggio y Powel (1999), el que en una primera etapa buscaba dar cuenta de la homogeneidad de las formas y las prácticas, evidenciando la interdependencia compleja entre y dentro de los campos organizacionales. Sobre este punto, consideramos que el Convenio Colectivo de Trabajo para los docentes de las instituciones universitarias nacionales constituye en un mecanismo isomórfico institucional, al establecer un marco legal general y promover varios de los lineamientos comunes para las CD/CA.

No obstante, ampliaciones analíticas posteriores reformulan la noción al afirmar que las restricciones en el ambiente no sólo limitan, sino que también abren posibilidades de cambio (Powell, 1999). De allí que se reconozca la existencia de una amplia gama de influencias institucionales y de respuestas internas, donde cobran centralidad las estrategias de los actores. En esa dirección, analizamos los diseños institucionales de CD/CA en busca de la diversidad propia de los 
procesos de organizacionales de implementación, donde los actores despliegan modalidades flexibles de interacción, colaboración y negociación en relación con las reglas.

De este modo, el enfoque aquí adoptado privilegia entonces los aspectos institucionales del cambio, con el propósito de identificar transformaciones o "isomorfismos" en la implementación de los diseños de CD/CA en las universidades nacionales, entendidas desde un plano de análisis organizacional (Powell y DiMaggio 1999).

En relación con la estrategia metodológica, como se mencionó anteriormente, la investigación es de corte exploratorio y se nutre del relevamiento de las normativas que regulan la carrera docente en seis casos seleccionados, en el ámbito de las universidades nacionales' . Se trata de un estudio sobre los "diseños institucionales" de la CD, donde se prioriza una estrategia documental y un abordaje institucional a fin de identificar cambios en los diseños de CD/CA, como así también lineamientos comunes y tendencias prevalecientes en los procesos de diseño de las reglas que orientan a la profesión académica. En esta instancia, no avanzamos en el estudio de los procesos de implementación de los diseños, aunque reconocemos la importancia que adquiere el análisis de las reglas no escritas para comprender en investigaciones futuras las prácticas cotidianas que dan sentido al orden institucional. Asimismo, revisar la normativa nos permite aproximarnos a nuevos acuerdos colectivos que tienen lugar en las universidades públicas argentinas, orientados por la influencia de las ideas de política, y que aspiran tanto a modificar las reglas que ordenan a la universidad como a la docencia universitaria en tanto profesión.

\section{revisar la normativa nos permite aproximarnos a nuevos acuer- dos colectivos que tienen lugar en las universidades públicas argentinas, orientados por la influencia de las ideas de política, y que aspiran tanto a modificar las reglas que ordenan a la uni- versidad como a la docencia universitaria en tanto profesión.}

\section{DISEÑOS INSTITUCIONALES EN ACCIÓN}

Al analizar los diseños institucionales de CD observamos dos tendencias generales en relación con los objetivos de la evaluación. La primera se orienta a generar la estabilidad y promoción en la situación y condición laboral de los docentes universitarios (De Miguel, 1998), por lo que la evaluación adopta funciones acumulativas y supone la definición de estrategias apropiadas para contar con información que colabore con la toma de decisión en cada uno de los peldaños de la carrera (Araujo, 2013). En la segunda tendencia, la evaluación es considerada una herramienta para la mejora institucional y académica, en la medida en que favorece el diseño de estrategias que influyan en la mejora de la actividad docente y el desarrollo profesional (Araujo, 2013). Por lo tanto, adquiere una función más formativa, en la medida 
que prevé instancias y dispositivos que les permitan a los docentes universitarios reflexionar sobre su desempeño y sus prácticas. Con respecto a la organización, las CD/CA presentan un abanico diverso de diseños donde en un extremo encontramos aquellos centralizados a nivel institucional, de corte más reglamentarista mientras que en el otro, los más descentralizados con breve apartados, donde las mayores precisiones terminan de definirse y negociarse en el ámbito de las distintas unidades académicas (Rovelli, De la Fare y Lenz, 2016). De todas formas, tanto en el plano de la evaluación como en el de la organización de las CD/CA, identificamos modelos híbridos que combinan o mixturan elementos de ambas tendencias y/o modelos organizativos.

En adelante, presentamos el análisis de las cinco dimensiones de estudio: acceso, permanencia y promoción; funciones, categorías y trayectorias docentes; conformación de las comisiones evaluadoras y las modalidades y dispositivos de evaluación.

Acceso, permanencia y promoción

Con respecto al acceso, observamos en los diseños institucionales un acuerdo generalizado en que se realiza por concurso público y abierto de antecedentes y oposición. El Convenio Colectivo de Trabajo (CCT) también avala esta condición para el acceso e incorpora la posibilidad de contar con un veedor gremial durante esa instancia.

Por su parte, la permanencia en la CD/CA se rige por sistemas de evaluación que combinan distintas modalidades y periodicidades. Del análisis de las normativas, surgen dos dispositivos principales de permanencia: a) los que contemplan exclusivamente evaluaciones por periodos para revalidar los cargos y b) los que combinan evaluaciones docentes anuales de carácter general y evaluaciones por períodos para revalidación. En cuanto a la periodicidad, en las normativas analizadas, la evaluación se estipula desde los 3 a los 6 años, con amplias variaciones entre las instituciones. EI CCT se orienta especialmente a generar estabilidad a través de las evaluaciones periódicas para revalidar la permanencia en el cargo, las cuales recomienda que se realicen "cada cuatro años o en un tiempo mayor según lo establecido en la reglamentación de cada Institución Universitaria Nacional" (art. 12). Llama la atención que en el diseño de una CD las evaluaciones periódicas de desempeño abarcan tanto a los docentes ordinarios como interinos de la Universidad.

En cuanto a la promoción, en algunos casos los diseños contemplan tanto el ascenso de categoría como el cambio en la dedicación; otras universidades, en cambio, circunscriben la promoción al ascenso de categoría inmediatamente superior. En forma unánime, los docentes ordinarios y/o concursados son los que tienen derecho de acceder a la promoción. En líneas generales, ésta adopta dos modalidades diferenciadas: por evaluación de desempeño (ascenso por dispositivos de evaluación presenciales y no presenciales); y por concurso público y abierto de antecedentes y oposición. Al respecto, el CCT liga el ascenso en los distintos cargos de la carrera a la realización de un concurso público y abierto de antecedentes y oposición. Sólo la promoción al cargo 
de Jefe de Trabajos Prácticos se establece por concurso público, cerrado, de antecedentes y oposición. En este punto, el CCT deja un margen mayor a los acuerdos institucionales que validen la promoción, dado que los sujeta a la "disposición estatutaria o acuerdo paritario local que establezca otro mecanismo más beneficioso para el docente".

No obstante, en los diseños institucionales pueden convivir ambos modelos, discriminados según los cargos y/o las dedicaciones. Nos referimos al concurso circunscripto a un solo docente o bien instancias internas o cerradas de promoción, en las que Jefes de Trabajos Prácticos y Profesores Asociados pueden solicitar el concurso para acceder a los cargos de Profesores Adjuntos y Titulares. Por otra parte, otros diseños institucionales asocian directamente la posibilidad de promoción a una convocatoria especial supeditada a la disponibilidad presupuestaria. Mientras que algunas normativas más innovadoras vinculan la promoción de categoría docente a un proceso basado en la postulación voluntaria de los docentes, donde la universidad garantiza la apertura del proceso de categoría aun cuando el cargo no cuente con asignación presupuestariaii . En este caso, para acceder al ascenso de categoría se debe acreditar un mínimo de seis años como Profesor Ordinario, en la categoría inmediatamente inferior y haber obtenido "aprobado" en las dos evaluaciones de desempeño.

\section{FUNCIONES, CATEGORÍAS Y TRAYECTORIAS DOCENTES}

Existe una tendencia hacia la diferenciación de funciones y trayectorias; en particular entre la docencia y la investigación. El argumento central que se sostiene es que es responsabilidad de las universidades desarrollar y definir distintas funciones pero que éstas no deberían recaer en un mismo sujeto sino ser coordinadas y articuladas institucionalmente.

\section{Existe una tendencia hacia la diferenciación de funciones y tra- yectorias; en particular entre la docencia y la investigación. El argumento central que se sostiene es que es responsabilidad de las universidades desarrollar y definir distintas funciones pero que éstas no deberían recaer en un mismo sujeto sino ser coor- dinadas y articuladas institucionalmente.}

Es más, aquellos diseños que promueven la fijación de trayectorias diferenciadas argumentan que el perfil y la productividad son de diferente naturaleza, por lo que resulta infrecuente que un mismo académico reúna la excelencia en todas las funciones sustantivas universitarias (Garritz Ruiz, 2011). A su vez, se plantea que no debería haber distinción entre ambas trayectorias en cuanto a la posición, las remuneraciones o las expectativas del servicio universitario (Garritz Ruiz, 2011). Otros autores reconocen la importancia de la valoración integral del trabajo académico, pero contemplan la posibilidad de in- 
corporar interfaces con otras actividades como la investigación y la extensión y transferencia a lo largo de la trayectoria académica e institucional. De todas formas, advierten sobre la dificultad de conjugar, en un mismo académico y con la misma intensidad y calidad, las tres actividades centrales de la universidad. Incluso en algunos trabajos se señala que la evaluación de todas las actividades, además de la selección de las más ponderadas en el proceso, suele provocar la intensificación del trabajo académico en lugar de la profesionalización docente (Araujo, 2013).

Los diseños de $C D$ analizados revelan que las universidades evalúan a sus docentes a partir de distintas funciones y actividades universitarias: docencia (o docencia y formación); investigación (a veces asociada a: desarrollo, innovación socio-productiva, vinculación y transferencia, etcétera); vinculación y transferencia; extensión (a veces asociada a servicios, transferencia, etcétera); evaluación y gestión (a veces denominada función institucional o asociada a representación $\mathrm{y}$ /o gobierno). Mientras que el CCT reconoce a la enseñanza, investigación, extensión y formación como funciones generales, en los diseños institucionales surgen variantes y nuevas actividades. Por otra parte, algunos regímenes de CD/CA establecen funciones por categoría y dedicación, por lo que definen articulaciones variables para los docentes. En ese sentido, en el diseño de una CD se establece que para los cargos de dedicación exclusiva y semiexclusiva es condición necesaria, más no suficiente, que junto con el módulo de docencia el de investigación científica, creación artística y/o innovación tecnológica o extensión sea calificado como satisfactorio. A su vez, para los cargos de dedicación simple, en la evaluación del módulo docencia se considera especialmente la actualización académica del evaluado.

Sin embargo, algunas casas de estudio acuerdan evaluar a sus docentes a través de perfiles o trayectorias más específicas, como docencia; docencia y desarrollo profesional; docencia e investigación; docencia y extensión. En otros casos, queda librado a la autonomía de las unidades académicas definir con "cada docente la combinación de funciones para el cumplimiento de su tarea", mientras que dicha combinación deberá incluir a la función docente. En la misma dirección, otra CD propone como condición necesaria, más no suficiente, que la evaluación correspondiente el módulo docencia sea satisfactoria para que la instancia en su conjunto se considere aprobada.

\section{CONFORMACIÓN DE LAS COMISIONES EVALUADORAS}

A fin de analizar la conformación de las Comisiones Evaluadoras, adoptamos dos criterios: uno político, asociado a la participación de los claustros y otro de corte organizacional, vinculado a la presencia de la disciplina o la institución.

En cuanto a la dimensión política, el análisis de los casos seleccionados permite identificar tres tipos de diseños: en primer lugar, algunos preservan la conformación tripartita de los jurados de los concursos 
públicos y abiertos. Allí están presentes con una participación plena los docentes que conservan la mayoría de la representación, los estudiantes y los graduados, siendo el esquema de representación más común el de tres, uno y uno respectivamente. En segundo lugar, existen diseños que le otorgan una participación plena a los docentes en la evaluación del conjunto de las funciones que se desarrollan (docencia, investigación, extensión y/o transferencias, entre otras), donde los estudiantes tienen una participación más restringida y centrada en la evaluación de la docencia únicamente; mientras que los graduados podrían incluso participar como veedores. En tercer lugar, otros diseños consideran como actores legítimos y exclusivos para participar en la evaluación de los docentes a sus propios pares.

En el análisis de la dimensión organizacional, es posible reconocer diferentes diseños que priorizan más a la disciplina o la institución y que definen un criterio de corte endógeno o exógeno para la composición de los profesores miembros del jurado. En un extremo, se conforman comisiones con evaluadores externos a la institución; mientras que en el otro, los evaluadores forman parte de las instituciones, por lo que en el primero se prioriza a la disciplinas y en el segundo, a la institución. De un modelo a otro, es posible identificar casos donde la mayoría de los evaluadores deben ser externos a la institución y se privilegia un criterio disciplinar para su convocatoria. Otros formatos definen que uno de los profesores debe ser de la propia facultad, otro externo a la unidad académica, pero de la misma universidad y un tercero externo a la institución. En estos esquemas, los criterios de la disciplina y de la institución se entrecruzan para construir los juicios sobre el desempeño de los docentes universitarios.

La regulación del ingreso a carrera en el CCT (Capítulo III, art. 11) prioriza un esquema que asegura la participación de los pares en la evaluación docente, por lo que legitima la experiencia profesional de profesores que se desenvuelven en la misma área de conocimientos que el evaluado. No obstante, en este punto, son los diseños institucionales analizados los que recuperan la conformación tripartita del cogobierno universitario, la que en general se observa también en los reglamentos de concursos. Como novedad, el CCT establece que cada una de las asociaciones sindicales docentes con personería o inscripción gremial en el ámbito de actualización en las instituciones universitarias nacionales en las que se lleve adelante el concurso, podrá designar un veedor gremial. Esta incorporación refleja la relevancia que tuvo el actor sindical en la homologación del CCT.

\section{MODALIDADES Y DISPOSITIVOS DE EVALUACIÓN}

Del relevamiento realizado surge que algunos diseños de evaluación priorizan modalidades e instrumentos orientados a generar condiciones para el mejoramiento de la práctica del docente universitario; mientras que otros adoptan rasgos más burocráticos que los distancian de la posibilidad de promover prácticas más sustantivas. 
algunos diseños de evaluación priorizan modalidades e instrumentos orientados a generar condiciones para el mejoramiento de la práctica del docente universitario; mientras que otros adoptan rasgos más burocráticos que los distancian de la posibilidad de promover prácticas más sustantivas.

En esa dirección, identificamos diseños que requieren de un encuentro presencial entre el jurado y el docente evaluado hasta dispositivos, aunque menos frecuentes, donde el docente sólo debe completar sus antecedentes a través de una aplicación web. Otros regímenes combinan instancias presenciales y no presenciales, las que puede variar como resultado de un dictamen negativo o bien ante una solicitud de promoción.

Un rasgo novedoso es que las Comisiones Evaluadoras cuentan con diferentes informes de diferentes actores sobre el desempeño del docente evaluado. En general, las normativas establecen que los evaluadores contarán con una declaración del propio docente sobre la labor realizada, como así también con evaluaciones de otros actores institucionales o externos a la universidad. Asimismo, el docente debe presentar el plan de actividades que se propone desarrollar. En suma, las comisiones evalúan los antecedentes académicos del postulante; los diferentes informes elaborados por las distintas instancias de gestión $y / o$ actores institucionales $y$ el plan de actividades en docencia, investigación, extensión y transferencia, a lo que en algunos casos se suma la formación de recursos humanos y el compromiso institucionaliii .

Otro elemento a destacar es que en todos los diseños institucionales analizados se incorpora una encuesta a estudiantes, en tanto instrumento de evaluación del desempeño docente. De este modo, las percepciones estudiantiles sobre el proceso de enseñanza y de aprendizaje cobran un papel central en los nuevos modos de regular la práctica docente universitaria. En cuanto a las pruebas de oposición, se evidencian cambios importantes, al ser desplazada paulatinamente la clase pública por otras modalidades de evaluación, como la entrevista y el coloquio. En estas instancias se suelen considerar aspectos vinculados a la actualización del postulante en el campo de conocimiento específico y a su desempeño académico o bien en otros casos, cuestiones ligadas a la planificación de actividades de docencia, investigación y/o extensión e incluso a las tareas que impliquen un compromiso institucional.

\section{CONSIDERACIONES FINALES}

El análisis de los diseños institucionales de CD/CA en las universidades nacionales seleccionadas muestra distintos propósitos en los sentidos de la evaluación que abarcan desde objetivos de estabilidad y regulación del trabajo académico hasta lógicas que involucran el mejoramiento de los procesos de formación y desempeño de los do- 
centes, con incidencia en el plano de las políticas académicas e institucionales en su conjunto.

\section{El análisis de los diseños institucionales de CD/CA en las univer- sidades nacionales seleccionadas muestra distintos propósitos en los sentidos de la evaluación que abarcan desde objetivos de estabilidad y regulación del trabajo académico hasta lógicas que involucran el mejoramiento de los procesos de formación y des- empeño de los docentes, con incidencia en el plano de las políti- cas académicas e institucionales en su conjunto.}

A su vez, si bien existe cierta amplitud en las modalidades organizativas que los moldean como así también en los criterios que rigen la permanencia y promoción, los diseños institucionales presentan un alto consenso en términos del acceso a la CD, en sintonía con lo establecido en el CCT. En este punto, el concurso público y abierto de antecedentes y oposición continúa siendo el mecanismo privilegiado de ingreso. No obstante, la clase pública y la exposición erudita de conocimiento parece desdibujarse ante los dispositivos de evaluación más vinculados a la actualización docente, planificación y desempeño académico y al compromiso institucional.

En respuesta al interrogante sobre el sentido del cambio en los diseños de CD/CA en las organizaciones, tanto la homologación del primer Convenio Colectivo de Trabajo (CCT) como la circulación de expertos, autoridades y representantes en los debates sobre la orientación de las normativas, junto con la difusión de experiencias novedosas de implementación de CD/CA en el subsistema universitario, se traducen en ciertos diseños miméticos que generan una mayor homogeneización de las CD/CA implementadas. Ante estas tendencias de cambio, también encontramos una mayor diversidad en los procesos organizacionales de implementación de diseños de la carrera, como resultado de la importancia del tiempo, las instituciones y sus actores, en un subsistema universitario que se asienta en la autonomía institucional y que resulta más complejo y diferenciado que en el pasado.

Con todo, el análisis de los diseños de CD permite tanto identificar algunas tendencias generales en la configuración actual de la docencia universitaria, así como también reconocer cambios y orientaciones novedosas en los sentidos de la evaluación y la orientación de la profesión académica. Asimismo, esperamos contribuir a un debate más amplio en torno al problema de las dinámicas y orientaciones que asumen los cambios en las universidades desde el análisis de las políticas y la perspectiva de las instituciones y sus actores. 


\section{BIBLIOGRAFIA}

Araujo, S. (2013). "Evaluación de la docencia en la universidad: cuestiones y sentidos en la definición de una propuesta". Ponencia presentada en el VII Encuentro Nacional y IV Latinoamericano de la Universidad como objeto de investigación, San Luis: UNSL.

De Miguel Díaz, M. (1998). "La evaluación del profesorado universitario. Criterios y propuestas para mejorar la función docente". Revista de Educación $\mathrm{N}^{\circ}$ 315, 67-85. Recuperado de: http://www.educacionyfp.gob.es/dam/jcr:bb417012-8e71-408f-b446-3a5d7d1bc10b/re3150400463-pdf.pdf

Di Maggio, P. y Powell, W. (1999). "Introducción". En Powell, W. y P. Di Maggio (eds.). El nuevo institucionalismo en el análisis organizacional, México: Fondo de Cultura Económica.

Fontaine, G. (2015). “Lecciones de América Latina sobre las dimensiones racionales, cognitivas e institucionales del cambio de políticas". Íconos Revista de Ciencias Sociales, No. 53, Vol. 19, 11-30, doi:10.17141/iconos.53.2015.1509.

Garritz Ruiz, A. (2011). "Reflexiones sobre dos perfiles universitarios: el docente y el investigador". Publicaciones Anuies, № 102, Vol. 26, 1-9, México, D.F. Recuperado de: http://publicaciones.anuies.mx/pdfs/revista/Revista102_ S1A1ES.pdf

Powell, W. (1999). “Expansión del análisis institucional". En Powell, W. y P. Di Maggio (eds.). El nuevoinstitucionalismo en el análisis organizacional, México: Fondo de Cultura Económica.

Rovelli, L., De la Fare, M. y Lenz, S. (2016). "Expansión y nuevas configuraciones en los regímenes de Carrera Académica/docente en las universidades nacionales de la Argentina". Revista Argentina de Educación Superior (RAES), núm. 12, 162-178. Recupe

\section{NOTAS}

\footnotetext{
iSe toman en consideración las CD/CA de la Universidad Nacional de Rosario, Córdoba, del Centro de la Provincia de Buenos Aires, Mar del Plata, General Sarmiento y Quilmes.

ii En ese caso, la institución designa al docente en la categoría obtenida y garantiza que el cargo en cuestión cuente con asignación presupuestaria en un plazo no mayor de tres años, contados a partir de la fecha de la nueva designación.

iii En líneas generales, en los diseños analizados, se solicitan informes sobre el desempeño del docente al responsable de la materia, cátedra, área o departamento y sobre la situación académico-institucional del postulante a la secretaría académica. En algunos casos, se incluyen informes de actividades realizadas por el propio postulante y también, por los directores de los proyectos de investigación y/o extensión en lo que haya participado durante el períodos.
} 